\title{
An Investigation of the Proximity Effect of Millimeter-Wave MMICs in Flip-Chip Configuration
}

\author{
Oliver Nagy ${ }^{1}$, Patric Heide ${ }^{2}$, Andreas Springer ${ }^{3}$, Robert Weigel ${ }^{3}$ \\ ${ }^{1}$ Siemens AG, Corporate Technology, D-81730 Munich, Germany, email: \\ oliver.nagy@ieee.org, Phone: ++ 4989636 48230, Fax: ++ 498963646881 \\ ${ }^{2}$ EPCOS AG, D-81617 Munich, Germany \\ ${ }^{3}$ University of Linz, A-4040 Linz, Austria
}

\begin{abstract}
A comparison between flip-chip mounting of monolithic microwave integrated circuits (MMICs) in microstrip transmission line technology and coplanar transmission line technology is presented. The influence of substrate proximity effects on the electrical behavior of the MMICs is examined and illustrated in normalized frequency independent graphs. Based on the results of this work, the performance of microstripMMICs used in flip-chip configuration can be estimated as a function of the chosen assembly geometry.
\end{abstract}

\section{INTRODUCTION}

Flip-Chip Mounting Technology (FCMT, Fig. 1) in the millimeter wave range became popular in the last few years, since it offers significant electrical performance advantages [1].
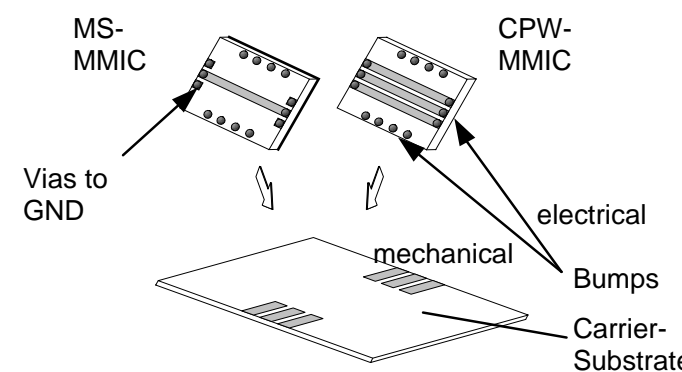

Fig. 1. Flip-Chip Mounting Technology.

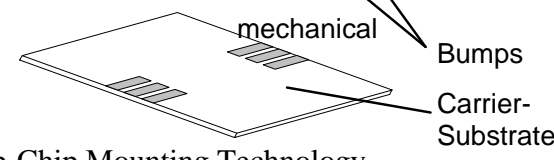

The common opinion is that MMICs designed in coplanar transmission line technology (coplanar waveguide, CPW) have a high compatibility with flip-chip mounting technology, while microstrip (MS) MMICs are not compatible. Various studies in the past have demonstrated design rules for finding the optimum bump height, distance or shape [2, 3] in order to minimize the transition loss. In this paper, the proximity effect corresponding to the distance between chip and substrate is examined for both microstrip and coplanar MMICs. A variation of the distance of the MMIC to the substrate in the FCMTprocess can be realized by choosing different heights of the bumps.

Fig. 2 shows the distribution of the electrical field lines in the MS- (left) and CPW- (right) MMIC FCMT-configuration. The influence of the substrate is obvious.

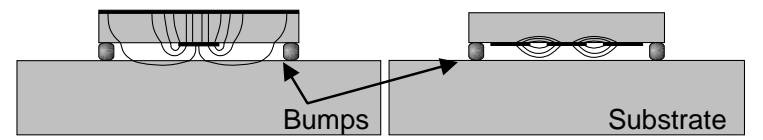

Fig. 2. Electrical field of a microstrip and a coplanar MMIC flip-chip mounted.

\section{GENERAL DESCRIPTION OF ANALYSIS}

The narrow gap between the substrate and the MMIC surface causes an increase of the relative dielectric constant $\varepsilon_{r}$ and of the characteristic impedance $Z_{C}$ of the transmission line on the MMIC. The consequence is an impedance mismatch of the transistors on the chip and a mismatch at the ports of the MMIC. This causes an additional insertion loss and a phase shift induced by the change in $\varepsilon_{r}$.

Both parameters have been determined with the commercial 3D-EM simulator Agilent HFSS ${ }^{\text {TM }}$ 5.6. The reference values for the transmission lines without a substrate influence has been determined with the software tool Agilent $\mathrm{ADS}^{\mathrm{TM}} 1.5$ LineCalc ${ }^{\mathrm{TM}}$.

Realistic values for the bump height are between 10 and $40 \mu \mathrm{m}$, higher values give an estimation of the chip behavior without substrate.

\section{BEHAVIOR OF MICROSTRIP MMICS}

The microstrip transmission line theory is thoroughly treated. The known formulas for the characteristic impedance obtained by different analysis methods are listed in [4]. To include a variation of the relative dielectric constant $\varepsilon_{\mathrm{r}}$ by the FCMT (in form of the distance $(d)$ chip - substrate) a new function $\sqrt{ } e_{M S}(d, f)$ is inserted in the general form of the equation for the characteristic impedance:

$$
Z_{C, M S}=\frac{f(h, W)}{\sqrt{\varepsilon_{r}} \cdot \sqrt{e_{M S}(d, f)}},
$$

where $f$ is the frequency and $f(h, W)$ is a function of the height $h$ of the substrate and of the width $W$ of the microstrip line.

$$
\sqrt{e_{M S}(d, f)}=\frac{\varphi_{M S}(d, f) \cdot c}{2 \cdot \pi \cdot l_{m} \cdot f \cdot \sqrt{\varepsilon_{r, e f f}}}
$$


with:

$\begin{array}{ll}\varphi(f) & \cdots \\ c & \cdots \\ l_{m} & \cdots \\ f & \cdots \\ \sqrt{\varepsilon_{r, e f f}} & \cdots\end{array}$

phase shift

velocity of light

mechanical length

frequency

effective permetivity

The $\varepsilon_{r, e f f}$ for the MS-line is:

\begin{tabular}{c|c}
$f[\mathrm{GHz}]$ & $\varepsilon_{r, \text { eff }}$ \\
\hline 77 & 8.710 \\
60 & 8.528 \\
38 & 8.310
\end{tabular}

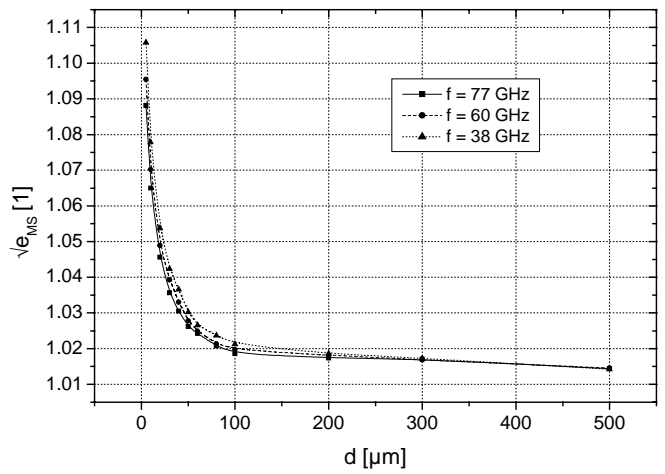

Fig. 3. $\sqrt{ } e_{M S}$ as a function of the distance $d$.

This dependence of $\sqrt{ } e_{M S}(d, f)$ results in an additive insertion loss $\Delta\left|S_{21}\right|$, calculated in $[\mathrm{dB} / \mathrm{mm}]$, see Fig. 4 , and for the phase shift, Fig. 5 , in $[\% \mathrm{~mm}]$.

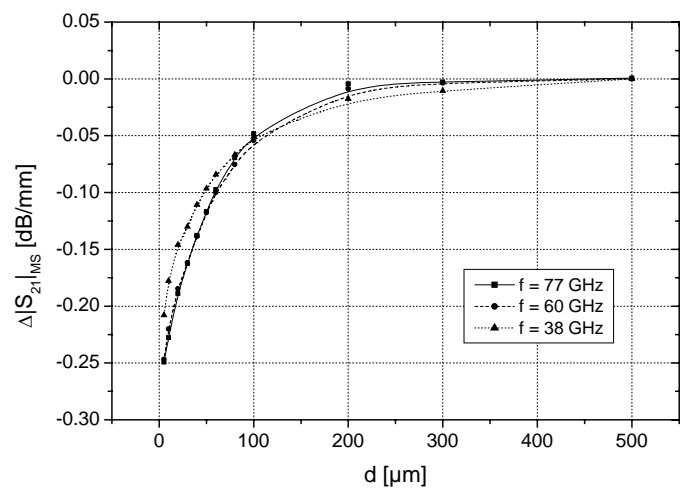

Fig. 4. Additional insertion loss of MS-MMIC.

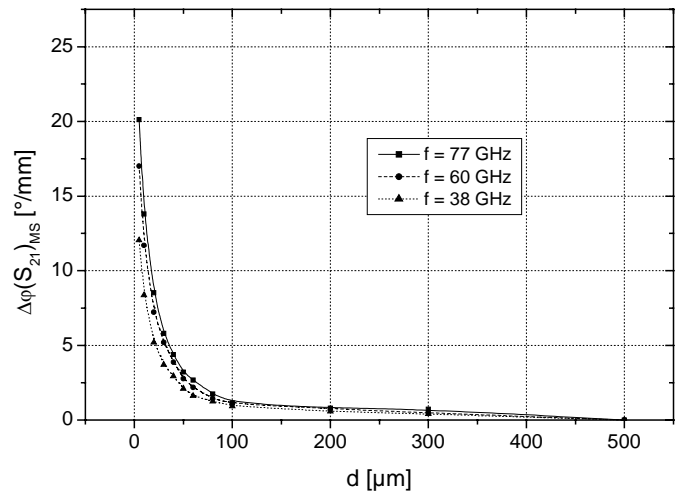

Fig. 5. Phase shift $\Delta \varphi\left(S_{21}\right)$ of MS-MMIC.

\section{BEHAVIOR OF COPLANAR MMICS}

For the equation of the characteristic impedance the general form is:

$$
Z_{C, C P W}=\frac{f(w, g, h)}{\sqrt{\varepsilon_{r}} \cdot \sqrt{e_{C P W}(d, f)}},
$$

$f(w, g)$ is a function of the width $w$ of the center conductor, the gap $g$ to the ground and the MMIC's height $h$. The function $\sqrt{ } e_{C P W}(d, f)$ is the same as in the MS case:

$$
\sqrt{e_{C P W}(d, f)}=\frac{\varphi_{C P W}(d, f) \cdot c}{2 \cdot \pi \cdot l_{m} \cdot f \cdot \sqrt{\varepsilon_{r, e f f}}}
$$

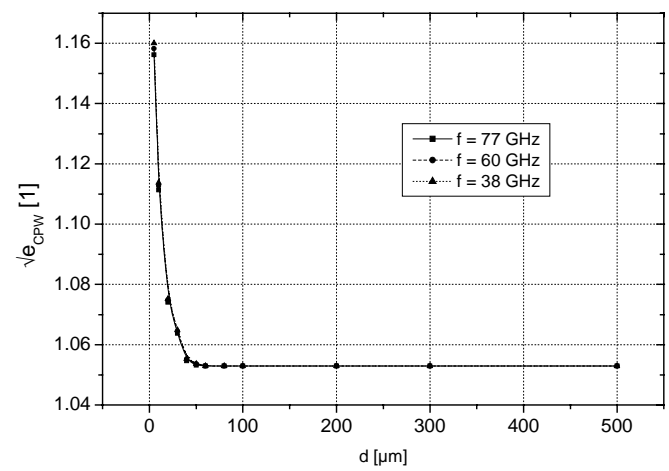

Fig. 6. $\sqrt{ } e_{C P W}$ as a function of the distance $d$.

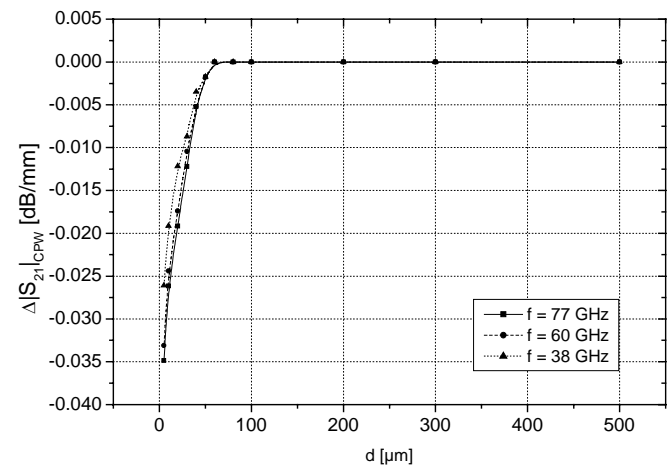

Fig. 7. Additional insertion loss of CPW-MMIC.

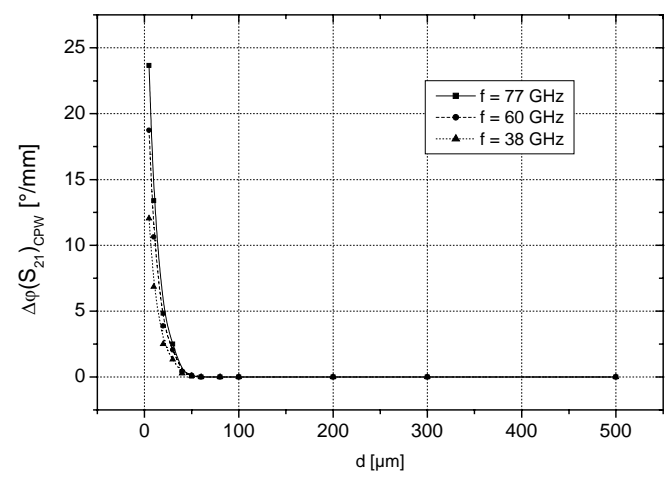

Fig. 8. Phase shift $\Delta \varphi\left(S_{21}\right)$ of CPW-MMIC. 
The equivalent graphs are for $\sqrt{ } e_{C P W}(d, f)$ Fig. 6, for the additional insertion loss Fig. 7 and for the phase shift Fig. 8. $\varepsilon_{r, \text { eff }}$ for the CPW line is 6.081.

\section{Simulation Results}

For a comparison of microstrip and coplanar MMIC behavior, it is advantageous to remove the frequency dependence in the previous equations. Fig. 911 show the normalized comparison graphs for both MS and CPW case, with Fig. 12-14 as the normalizing coefficients. The figures shown cover the realistic bump heights from 10 to $40 \mu \mathrm{m}$.

The equations (1) and (3) are enlarged to:

$$
Z_{C, M S}=\frac{f(h, W)}{\sqrt{\varepsilon_{r}} \cdot \sqrt{e_{\text {norm }, M S}(d)} \cdot \sqrt{e_{f, M S}(f)}}
$$

and

$$
Z_{C, C P W}=\frac{f(w, g, h)}{\sqrt{\varepsilon_{r}} \cdot \sqrt{e_{n o r m, C P W}(d)} \cdot \sqrt{e_{f, C P W}(f)}} .
$$

For the case of a MS and CPW chip the equations for $\sqrt{ } e_{f}(f) \quad((2)$ and (3)) have to be enlarged equivalently.

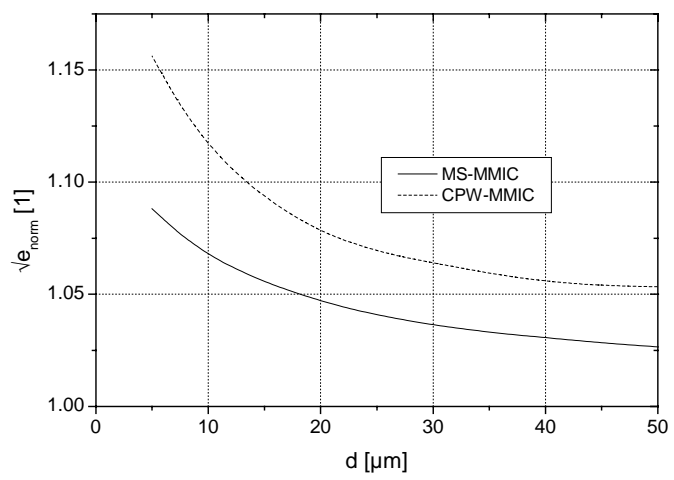

Fig. 9. $\sqrt{ } e_{\text {norm }}(d)$ of MS and CPW MMIC.

The graphs in Fig. 9 and Fig. 11 should be the same for both MMIC types due to the relative dielectric factor and the phase shift are only dependent of the position of the substrate. The slight difference observed is a measure of the computing accuracy, that is fairly high nevertheless.

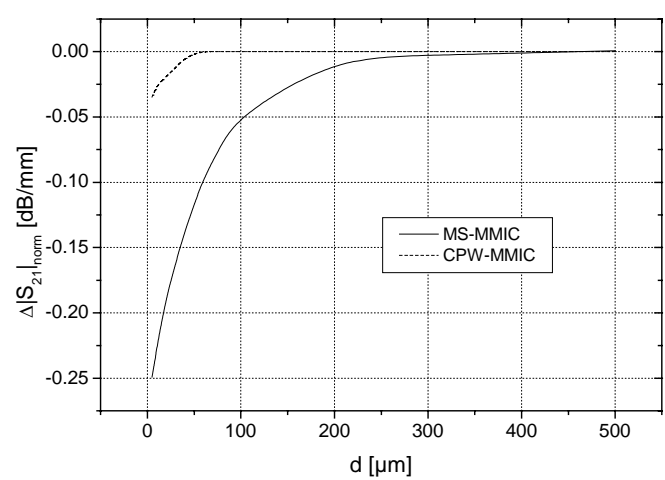

Fig. 10. Normalized additional insertion loss $\Delta\left|S_{21}\right|_{\text {norm }}(d)$.

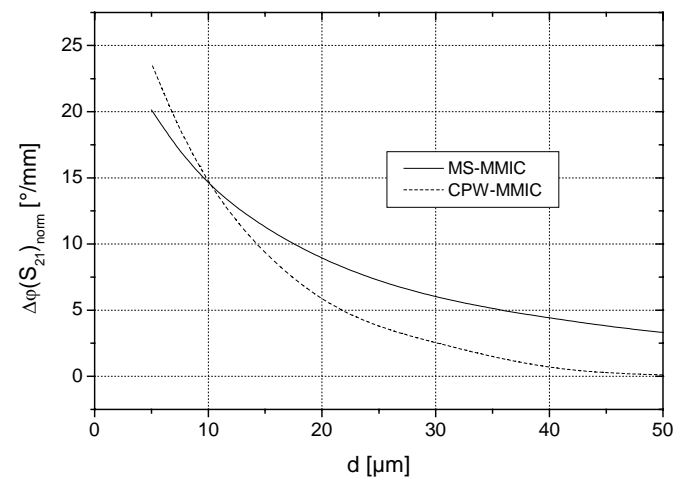

Fig. 11. Normalized phase shift $\Delta \varphi\left(S_{21}\right)_{\text {norm }}(d)$.

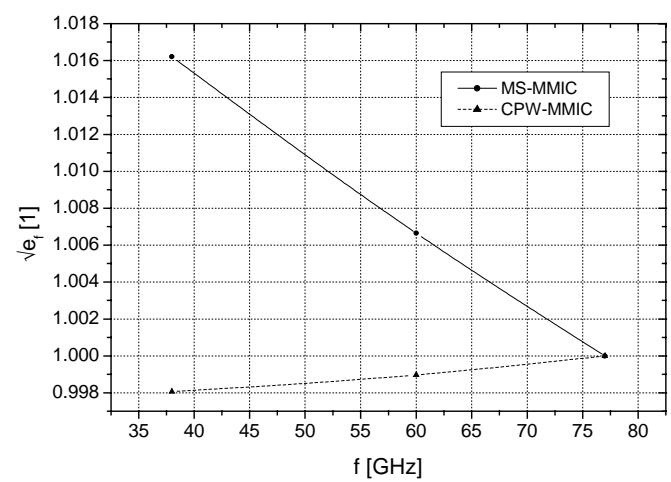

Fig. 12. Normalizing coefficient $\sqrt{ } e_{f}(f)$.

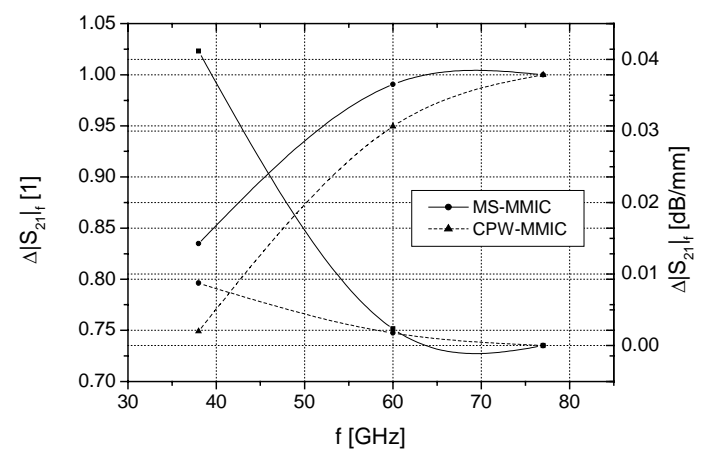

Fig. 13. Normalizing coefficient $\Delta\left|S_{21}\right| f(f)$. 


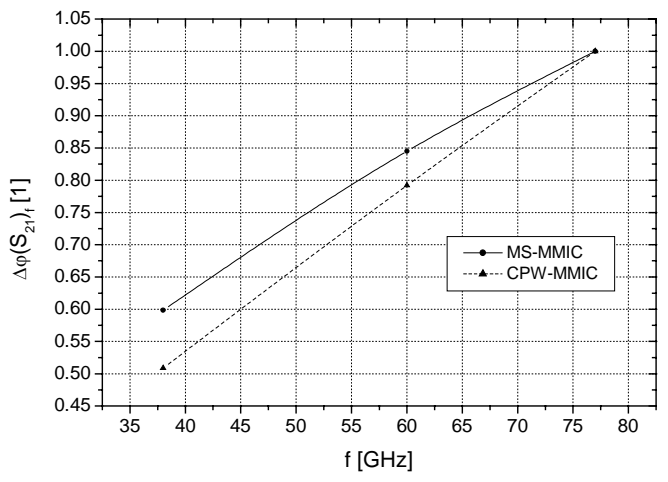

Fig. 14. Normalizing coefficient $\Delta \varphi\left(S_{21}\right)_{f}(f)$.

The frequency dependent parameters are obtained through

$$
\Delta\left|S_{21}\right|(d, f)=\Delta\left|S_{21}\right|_{\text {norm }}(d) \cdot \Delta\left|S_{21}\right|_{f}(f)
$$

in $[\mathrm{dB} / \mathrm{mm}]$ or

$$
\Delta\left|S_{21}\right|(d, f)=\Delta\left|S_{21}\right|_{\text {norm }}(d)+\Delta\left|S_{21}\right|_{f}(f)
$$

in $[\mathrm{dB} / \mathrm{mm}]$ from the right scale in Fig. 14 and

$$
\Delta \varphi\left(S_{21}\right)(d, f)=\Delta \varphi\left(S_{21}\right)_{n o r m}(d) \cdot \Delta \varphi\left(S_{21}\right)_{f}(f)
$$

in $[\% \mathrm{~mm}]$.

With respect to a successful design of flip-chip circuits these simulations provide a measure for the minimum bump heights as a function of the acceptable loss and phase shift. The results can be used as a design rule. Actual values for the bump height best suited for a specific MMIC are obtained by multiplying the readouts of the figures by the chip length in $[\mathrm{mm}]$.

\section{EXPERIMENTAL RESULTS}

The experimental verification of the simulated results is illustrated in Fig. 15. Two samples of the same MS-MMIC were first measured on chip. Afterwards, these chips were measured flip-chip mounted in two different configurations, the standard configuration as well as a configuration where the substrate proximity effect was actually removed by (laser-) drilling a radiation hole under the MMIC-area. A very good agreement with the previous graphs is given. The difference $\Delta\left|S_{21}\right|$ between on chip and flip chip measurements is the insertion loss of the bumps. This insertion loss matches the statements in [2] exactly.

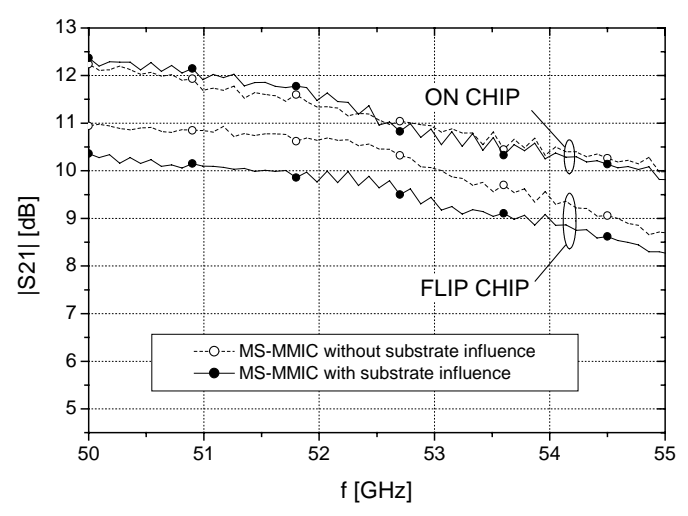

Fig. 15. Measurement with and without substrate influence.

Photographs of the flip chip mounted MS-MMIC are shown in Fig. 16.
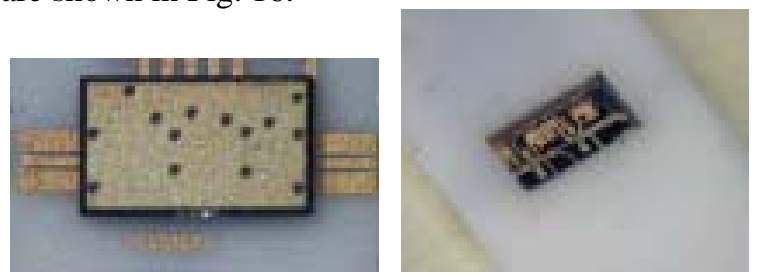

Fig. 16. Top (left) and bottom (right) view of flip chip (MS-) MMIC.

\section{CONCLUSION}

This paper has demonstrated theoretical and practical results that aid the successful design of flip-chip modules. Based on the applied theory and the corresponding simulation graphs a designer of a flip-chip circuit is able to quantitatively estimate the proximity effect between MMICs (both coplanar and microstrip) and the substrate. Therefore, this work provides some novel hints and design rules being required to achieve high performance flip-chip circuits in terms of insertion loss and phase shift.

\section{REFERENCES}

[1] P. Heide, A. Dabek, "Millimeter-Wave Module Technologies for Innovative Sensor and Communication Products", 2000 IEEE Int. Microwave Symposium, Boston, MA, Proc. Workshop WSK "Micro/Millimeter-Wave Transceivers for Mass Production: Design, Technology, Implementation".

[2] T. Krems, W. Haydl, H. Massler, J. Rudiger, "Millimeter-Wave Performance of Chip Interconnections Using Wire Bonding and Flip Chip", IEEE MTT-S Digest, pp. 247-50, 1996.

[3] Y.L. Wong, L. Patrison, D. Linton, "Flip Chip Interconnect Analysis at Millimetre Wave Frequencies", IEEE: Piscataway, NJ, USA, 1999, p. 82 -7 , iii+163 pp.

[4] K.C. Gupta, R. Garg, I. Bahl, P. Bhartia, "Microstrip Lines and Slotlines", Artech House, 1996. 